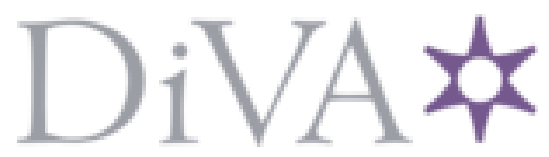

http://www.diva-portal.org

This is the published version of a paper presented at IEEE International Symposium on Personal, Indoor and Mobile Radio Communications (PIMRC).

Citation for the original published paper:

Tombaz, S., Zheng, Z., Zander, J. (2013)

Energy Efficiency Assessment of Wireless AccessNetworks Utilizing Indoor Base Stations.

In: Energy Efficiency Assessment of Wireless AccessNetworks Utilizing Indoor Base Stations (pp. 1-6). IEEE

N.B. When citing this work, cite the original published paper.

Permanent link to this version:

http://urn.kb.se/resolve?urn=urn:nbn:se:kth:diva-133930 


\title{
Energy Efficiency Assessment of Wireless Access Networks Utilizing Indoor Base Stations
}

\author{
Sibel Tombaz, Zhihao Zheng, and Jens Zander \\ KTH Royal Institute of Technology, Wireless@KTH, Electrum 229, 16440 Stockholm, Sweden \\ Email: sibel@kth.se, zhihao@kth.se, jenz@kth.se
}

\begin{abstract}
Energy efficiency in mobile radio networks has recently gained great interest due to escalating energy cost and environmental concerns. Rapidly growing demand for capacity will require denser and denser networks which further increase the energy consumption. In this regard, the deployment of small cells under macro-cellular umbrella coverage appears a promising solution to cope with the explosive demand in an energy efficient manner. In this paper, we investigate the impact of joint macro-and femtocell deployment on energy efficiency of wireless access networks, based on varying area throughput requirements. We take into account the the co-channel interference, fraction of indoor users, femto base station density and backhaul power consumption. It is shown that utilizing indoor base stations provide significant energy savings compared to traditional macro only network in urban areas with medium and high user demand where the gain increases up to $\mathbf{7 5}$ percent as more data traffic is offloaded to femtocells.
\end{abstract}

Index Terms-Energy Efficiency, Femtocells, Co-channel Deployment, Backhaul, Power Consumption.

\section{INTRODUCTION}

In recent years, operators are facing exponential growth in data traffic due to the rapid proliferation of smart-phones, laptops, and tablet PCs with built-in cellular access as well as flat rate tariff [1], [2]. This situation poses steeply reducing revenue per unit data consumed, and thus increases the significance of the cost effective solutions. Another important consequence of the data explosion is the rapid rise in energy consumption which is used to have minor impact on the operators operational expenditures (OPEX) [3], [4]. However, nowadays, energy bill constitutes 20-50 percent of the OPEX depending on number of off-grid sites, unit energy costs, etc [5]. To solve this issue, academia and industry are making great efforts to improve energy efficiency of the state-of-the-art wireless broadband networks at all levels [4], [6].

Considering the fact that exponential increasing demand for high data rates will require the deployment of several orders of magnitude more base stations (BSs), using high power macro BSs is expected to be neither energy efficient nor very sound from a radiation perspective. In this regard, heterogeneous networks (Hetnets), i.e., strategically located large number of small BSs such as micro-, pico- and femto BSs, under the macro-cellular umbrella coverage is believed to enable energy savings due to their low transmit power requirements [7], [8]. Especially femto BSs, i.e., low-power, low-cost, user-deployed base stations operating in licensed spectrum, have attracted great interest in order to address coverage and capacity needs in residental or enterprise environments in which 60 percent of voice and 70-90 percent of data traffic are expected to be originated [7], [9], [10]. However, co-channel operation with the existing macro-cellular network creates challenges due to the mutual interference generated between femtocells and macrocells. This issue has been widely investigated in the literature and the findings indicated that co-channel deployment has a minor impact on the performance of macro-cellular network with the appropriate configurations [11].

Moreover, the energy efficiency of different femtocell deployment architectures is analyzed in several papers [9], [12][14] in which contradictory conclusions are presented. In [15], the tradeoff between spectrum efficiency and energy efficiency is illustrated for joint macro-femto deployment where significant reduction in energy consumption per data delivered is illustrated with the cost of performance degradation due to co-channel interference. The large power savings via offloading data traffic to femtocells has also been presented in [13], [14], [16], considering urban areas with high user demand. On the contrary, despite its cost-effectiveness, joint macro-femto deployment is indicated to increase the energy consumption compared to traditional macro-only network for medium and high femto deployment densities and high number of supported users per macrocell [9]. This difference is claimed to be further increased when idle mode operation for macro BSs is implemented. The energy efficiency of user-deployed femto BSs are also compared with outdoor Hetnet deployment, i.e., macro-micro, and it is stated that these strategies have similar energy consumption for a given capacity enhancement requirement [12]. However, outdoor small cell deployment is proposed as a better solution for the operators due to their high convenience in terms of management and control [12].

There are two main reasons that the studies indicate contradictory conclusions. First, the power consumption of the network is modelled differently, i.e., transmit power only or total power consumption including idle and backhaul power consumption. Second, the energy efficiency comparison between different deployment strategies has been done either under a chosen performance constraint, e.g., average user throughput, network capacity, coverage, or only based on the total power consumption. However, in order to evaluate different solutions in the unbiased manner, network performance requirements by means of both coverage and capacity should be characterized.

In this paper, we assess the energy efficiency of joint macrofemto deployment with respect to area power consumption for a given coverage and area throughput requirement by considering backhaul power consumption which is often ignored in the literature. The results have been compared with traditional 
macro-only network offering service to both indoor and outdoor users and providing the same performance. Furthermore, we investigate the impact of several factors affecting area throughput and energy efficiency, such as femto BS density, the fraction of the indoor users, and inter site distance (ISD). The results show that energy efficiency improvement through joint macro-femto deployments are highly related to area throughput requirement and the amount of mobile traffic being offloaded to femtocells, where up to $75 \%$ power savings is found to be feasible in urban areas with high capacity demand.

The rest of the paper is organized as follows. In Section II, we introduce the system and power consumption models, and define the performance metrics. The simulation methodology and the results are presented in Section III and the last section concludes the paper.

\section{System Model And Definitions}

In this section, we introduce the system model which is used to analyze the impact of utilizing femto BSs on network energy efficiency, and define the performance metrics. We focus on downlink traffic which is the main consideration of network deployment.

\section{A. System Model}

In this paper, we consider a hexagonal grid of 19 sites as illustrated in Fig. 1, with a central reference cell surrounded by two tiers of interferers. Here, the term cell is used to indicate the hexagonal region of each site with inter site distance of $D=R \sqrt{3}$, which might be divided into several sectors.

In this layout, macro BSs with three sectors are deployed in the middle of the cell in a regular grid, transmitting through directional antennas, whereas femto BSs are equipped with a single omni directional antenna. Within the macro coverage area $\mathcal{A} \subseteq \mathbb{R}^{2}, N_{H}$ single floor houses of size $10 \mathrm{~m} \times 10 \mathrm{~m}$ are placed at random locations with a uniform distribution. In order to represent the fraction of the houses with a femto BS, we define a system parameter, $\rho_{p} \in[0,1]$ called femto BS penetration rate and deploy the femto BSs inside the houses accordingly. Here, $\rho_{p}=0$ represents the baseline system where all the users (indoor and outdoor) are served by macrocellular network whereas $\rho_{p}=1$ denotes the case in which each house has its own femto BS [17]. On the other hand, $I_{p} \in[0,1]$ is used to denote the the fraction of indoor users. Under the assumption that each house can simultaneously serve up to 6 users [17], [18], we randomly distribute the user over the reference cell based on the considered $I_{p}$. Those stay indoors are served by femto BSs if the house has a femto BS in order to increase the macrocell offloading benefits, otherwise they will be served by outdoor macro BSs.

To achieve universal frequency reuse, femtocells are considered to share the same bandwidth as the macro-cellular network, which is known as co-channel deployment. However, for simplicity, we assume that there is no coordination between macro and femto BS, nor between different femto BSs. On the other hand, for resource allocation, a simple round robin scheduling algorithm is used which allocates an equal part of the bandwidth to each active user without taking user channel conditions into account.

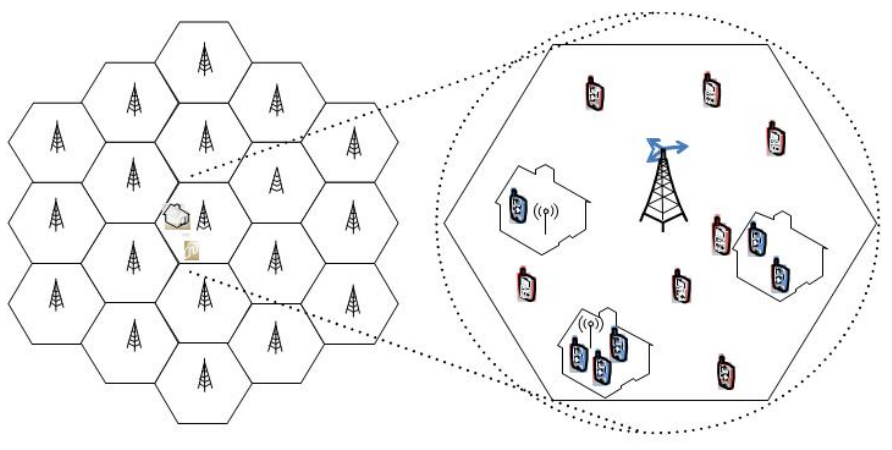

Fig. 1. Network Layout

\section{B. Propagation Model}

Received power at a terminal is affected by multiplication of three components which are; distance dependent path loss, shadowing and multipath. When we neglect the effect of multipath, received signal power can be written as below:

$$
P_{r x}[\mathrm{dBm}]=P_{t x}[\mathrm{dBm}]+G[\mathrm{~dB}]+A(\theta)-P_{L}[\mathrm{~dB}]-\psi[\mathrm{dB}]
$$

Here $P_{t x}$ is the base station transmit power per antenna, $G$ is the antenna gain, $P_{L}$ is the path loss and $\psi$ is the expected value for shadow fading. On the other hand, $A(\theta)$ denotes the transmitter array gain due to antenna pattern in $\mathrm{dBi}$ in the direction of $\theta,-180 \leq \theta \leq 180$ which is given by [19]

$$
A(\theta)=-\min \left[12\left(\frac{\theta}{\theta_{3 d B}}\right)^{2}, A_{m}\right]
$$

where $\theta_{3 d B}=70^{\circ}$ is the $3 \mathrm{~dB}$ beamwidth, and $A_{m}$ is the maximum attenuation. For omni directional antennas, $A(\theta)=0$. In this paper, we use 3GPP path loss models provided in [19] for urban deployment where path loss from user to macro BS is given by

$$
P_{L}(\mathrm{~dB})= \begin{cases}15.3+37.6 \log _{10} d, & \text { outdoor user } \\ 15.3+37.6 \log _{10} d+L_{\text {ow }}, & \text { indoor user }\end{cases}
$$

where $d$ is the distance between the user and macro BS in meters, and $L_{o w}$ is the penetration loss of an outdoor wall equal to $20 \mathrm{~dB}$. When the user is connected to femto BS, the path loss is calculated as [19]

$$
P_{L}(\mathrm{~dB})= \begin{cases}38.46+20 \log _{10} d+0.7 d_{2 D}, & \text { indoor user } \\ \max \left(2.7+42.8 \log _{10} d, 38.46+\right. & \\ \left.20 \log _{10} d\right)+L_{o w}+0.7 d_{2 D}, & \text { outdoor user }\end{cases}
$$

Here $0.7 d_{2 D}$ represents the penetration loss due to internal walls and $d_{2 D}$ is the distance inside the house. 


\section{Cell Coverage}

We define cell coverage as the fraction of the cell area where the received power is above a given level, $P_{\text {min }}$, i.e.,

$$
\mathcal{C}:=\frac{1}{|\mathcal{A}|} W \int_{\mathcal{A}} r \mathbb{P}\left[P_{r x} \geq P_{\min }\right] d_{r} d \phi
$$

In this paper we assume that femto BSs are deployed for capacity purposes and do not contribute to macrocell coverage.

\section{Area Throughput}

Let $\mathcal{I}_{\mathcal{M}}$ and $\mathcal{I}_{\mathcal{F}}$ represent the index set all sectors and femto BSs in the reference cell respectively. On the other hand, $\mathcal{I}_{\mathcal{A}}$, i.e., $\mathcal{I}_{\mathcal{M}} \cup \mathcal{I}_{\mathcal{F}}$, and $\mathcal{I}$, contain all the indices in the reference cell and the network. Since a mobile can only be served by one BS, area of the reference cell can be expressed as

$$
\mathcal{A}=\left(\bigcup_{i=1}^{\left|\mathcal{I}_{\mathcal{M}}\right|} \mathcal{A}_{m a, i}\right) \cup\left(\bigcup_{i=1}^{\left|\mathcal{I}_{\mathcal{F}}\right|} \mathcal{A}_{f e m, i}\right)
$$

where $\mathcal{A}_{m a, i}$ and $\mathcal{A}_{\text {fem,i }}$ represent the macro sector and femto cell areas, respectively. Here, sector area includes the set all the points where corresponding BS provides the highest average received power, excluding the indoor areas served by femto BSs. When a user locates at $x \in \mathcal{A}$ with respect to a BS $i \in$ $\mathcal{I}_{\mathcal{A}}$, spectral efficiency for ideal AWGN channel is expressed based on Shannon-Hartley theorem as shown below:

$$
r_{i}(x)=\min \left[\log _{2}\left(1+\gamma_{i}(x)\right), r_{\max }\right] \quad(\mathrm{bps} / \mathrm{Hz}) .
$$

Here, $r_{\max }$ reflects the maximum sustainable rate in practice and $\gamma_{i}(x)$ represents the signal to noise ratio (SINR) of the user in location $x$, i.e.,

$$
\gamma_{i}(x)=\frac{P_{r x, i}(x)}{\sum_{j \in \mathcal{I} \backslash\{i\}} P_{r x, j}(x)+\sigma^{2}},
$$

where $P_{r x, i}(x)$ is the received power from BS $i$ and $\sigma^{2}$ denotes the noise power.

Let $X_{i}$ denotes the random realization of the user positions in $\mathcal{A}$. Then, under a full buffer traffic model assumption, i.e., there is at least one mobile requesting data with all resources allocated, average area throughput can be written as

$$
\mathcal{T}:=\frac{1}{|\mathcal{A}|} W \sum_{i \in I_{A}} \mathbb{E}\left[r_{i}\left(X_{i}\right)\right], \quad\left(\mathrm{Mbps} / \mathrm{km}^{2}\right),
$$

where $W$ represents the system bandwidth.

\section{E. Area Power Consumption}

Here area power consumption is used as an energy efficiency metric which relates the total power consumed in the network to the corresponding network area, i.e., $\left|\mathcal{A}_{\text {net }}\right|=$ $19 \times|\mathcal{A}|$, which is measured in $\mathrm{W} / \mathrm{km}^{2}$ as below:

$$
\mathcal{P}_{\text {area }}=\frac{P_{\text {net }}}{\left|\mathcal{A}_{\text {net }}\right|} .
$$

Here $P_{n e t}$ denotes the total power consumption of the network which equals to sum of power consumption of the individual BSs in the network and the backhaul power consumption $\left(P_{b h}\right)$, which is given by

$$
P_{n e t}=\sum_{i \in \mathcal{I}} P_{i}+P_{b h}
$$

Due to consistency, we employ the power consumption model per sector, $P_{i}$, which is proposed in [20] for the considered base stations types as shown in the following expression:

$$
P_{i}= \begin{cases}a_{M} P_{t x}+b_{M} & \text { for a macro sector } \\ a_{F} P_{t x}+b_{F}, & \text { for a femto BS }\end{cases}
$$

Here, $P_{t x}$ denotes the power fed to the antenna. On the other hand, $a_{M}$ and $a_{F}$ represent the portion of the transmit power dependent power consumption due to feeder losses and power amplifier, whereas $b_{M}$ and $b_{F}$ account for the power consumption because of the active site cooling and the signal processing which constitutes the major part of the total power consumption of the BSs.

In this paper, in order to obtain consistent and realistic results to assess the impact of utilizing indoor base stations on energy efficiency, we incorporated fiber optic based backhaul power consumption into the analysis, which is given by [21]:

$$
P_{b h}=|\mathcal{I}| \times\left(b_{b h}+\frac{(1-\tau) P_{\text {switch }}^{\text {max }}}{n_{\text {ports }} C_{\text {switch }}^{\text {max }}} A g_{\text {switch }}+\frac{\tau P_{\text {switch }}^{\text {max }}}{n_{\text {ports }}}\right) .
$$

Here, $b_{b h}$ accounts for the power consumed by the backhaul transceiver, and the uplink interface, $P_{\text {switch }}^{\max }$ is the maximum power consumption of the switch and $A g_{\text {switch }}$ is the aggregate traffic traversing the switch. On the other hand, $\tau, C_{\text {switch }}^{\max }$ and $n_{\text {ports }}$ represent the percentage of the switch power that is independent of the network traffic, $\tau \in[0,1]$, maximum capacity of a switch and the number of ports of the switch, respectively. A more detailed explanation of these parameters can be found in [21].

It should be noted that here, power consumption of both femto and macro BSs are taken into account in order to comprehend environmental impact; despite the fact that energy cost of femto BSs belongs to home-owners rather than operators.

\section{Simulation Procedure and Results}

In this section, the performance of wireless access networks utilizing indoor base stations is evaluated in terms of area power consumption under coverage and capacity constraints. To this end, we first clarify the simulation setup and the methodology, and finally present the simulation results.

\section{A. Simulation Setup}

We consider a hexagonal grid of 19 sites as illustrated in Fig. 1, where inter site distance varied from $500 \mathrm{~m}$ to 1700 m. Within each macro cell, $N_{H}=30$ single floor houses of size $10 \mathrm{~m} \times 10 \mathrm{~m}$ are uniformly distributed. In addition, it is assumed that, with probability $\rho_{p}$, there is one femto BS deployed in the center of each house. We consider 60 active users per $\mathrm{km}^{2}$ in the network among which $100 \times I_{p}$ percent of 
TABLE I

Simulation AsSUmptions

\begin{tabular}{ll}
\hline \hline System and Path Loss Parameters & Value \\
\hline Frequency & $2 \mathrm{GHz}$ \\
Bandwidth & $5 \mathrm{MHz}$ \\
Antenna Gain (MBS/FBS) & $15 / 5 \mathrm{dBi}$ \\
Max BS transmit power (macro/femto) & $46 / 20 \mathrm{dBm}$ \\
Shadowing standard deviation (macro/femto) & $8 / 4 \mathrm{~dB}$ \\
Thermal Noise & $-174 \mathrm{dBm} / \mathrm{Hz}$ \\
Noise figure & $10 \mathrm{~dB}$ \\
Exterior wall loss & $20 \mathrm{~dB}$ \\
Min received power & $-90 \mathrm{dBm}$ \\
\hline Deployment and Model Specific Parameters & Value \\
\hline Inter site distance & $500-1700 \mathrm{~m}$ \\
User density per km ${ }^{2}$ (uniform) & 60 \\
Number of houses per macrocell & 30 \\
Max. spectral efficiency & $6 \mathrm{bits} / \mathrm{s} / \mathrm{Hz}$ \\
Femto penetration rate & {$[0,1]$} \\
Indoor user ratio & {$[0,1]$} \\
\hline Power Consumption Parameters & Value \\
\hline$a_{M} / a_{F}$ & $4.7 / 8$ \\
$b_{M} / b_{F}$ & $130 / 4.8 \mathrm{~W}$ \\
$b_{b h}$ & $3 \mathrm{~W}$ \\
$P_{\text {switch }}^{\text {max }}$ & $300 \mathrm{~W}$ \\
$\tau$ & 0.8 \\
$C_{\text {switch }}^{\text {max }}$ & $10 \mathrm{~Gb} / \mathrm{s}$ \\
$n_{\text {ports }}$ & 24 \\
\hline \hline & \\
\hline
\end{tabular}

them generated indoors. The selected user density represents an urban area where the average population density is around 1000 (citizens $/ \mathrm{km}^{2}$ ) whom are assumed to be served by three operators and average duty cycle of each mobile subscriber is $17.15 \%$ [18], [22].

The transmit powers of individual macro BSs are set based on Eq. (5) such that the received power at the cell edge is $P_{m i n}=-90 \mathrm{dBm}$ for a given inter site distance assuming an additional loss of $L_{o w}=20 \mathrm{~dB}$, whereas a fix transmit power of $20 \mathrm{dBm}$ is assumed for femto BSs. Power consumption model coefficients for the considered base station types are selected based on [20] and the power consumption of fiberoptic based backhaul, proposed in [21], is incorporated into the model to assess the area power consumption. In order to evaluate the spectral efficiency, we consider $r_{\max }=6 \mathrm{bit} / \mathrm{s} / \mathrm{Hz}$ in Eq. (7) with a full-buffer traffic model. The detailed assumptions on system and power consumption parameters are listed in Table I.

\section{B. Simulation Procedure and Results}

System level simulations are performed in order to analyze the achievable data rates for both indoor and outdoor users in the reference cell and to calculate the corresponding area power consumption values by considering different deployment strategies, i.e., macro only $\left(\rho_{p}=0\right)$, joint macro+femto $\left(\rho_{p} \in(0,1]\right)$. Here, for a given $\rho_{p}$, the houses with femto BSs are randomly chosen where each house is assumed to accommodate up to 6 users. It should be noted that sleeping mode operation for femto BSs has not been considered in this paper. Therefore, when a house with a femto BS does not have any user to serve, the BS is still assumed to consume the idle power, i.e., $P_{i}=b_{F}$. For all the

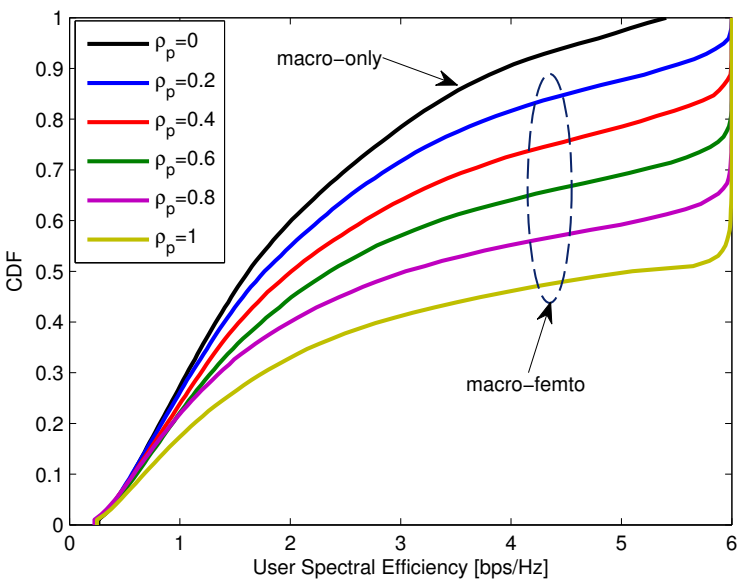

Fig. 2. CDF of spectral efficiency across users in the reference cell ( $I_{p}=0.5$, $\mathrm{R}=500 \mathrm{~m}, P_{\mathrm{tx}}^{\text {macro }}=46 \mathrm{dBm}, P_{\mathrm{tx}}^{\text {femto }}=20 \mathrm{dBm}$ )

considered deployment scenarios, we conduct 100 iterations with different user locations for a given inter site distance. Fig. 2 shows the cumulative distribution functions (CDFs) of spectral efficiencies across users in the reference cell for the range of $500 \mathrm{~m}$. It is clear from the figure that a significant improvement in user spectral efficiencies is achieved via femtocell deployment. This is due to the fact that indoor users enjoy much higher SINR because of shorter distance between the user and the serving BS as well as elimination of wall penetration loss. Besides, the additional interference because of co-channel deployment is observed to be minimal which is also demonstrated in [13].

In order to fairly assess whether utilizing femto BSs are beneficial in terms of energy efficiency compared to macroonly network, we compare the area power consumption of different deployment strategies under a certain area throughput and coverage requirements. By doing so, we prevent the cases where increase in total power consumption via excessive femtocell deployment constitutes a favorable choice for greener networks. To this end, we used the methodology proposed in [23] in order to define the optimal ISDs that minimize area power consumption of different topologies $\rho_{p} \in[0,1]$ for a certain target area throughput.

The methodology is based on the fact that there is an area power consumption minimizing ISD, $D_{k}^{*}$, for each strategy $k$, due to the tradeoff between the reduced transmit power to ensure fixed coverage and additional idle power consumption due to densification [1]. On the other hand, area throughput monotonically decreases with ISD where femtocell deployment provides significant gains. Under these circumstances, the methodology defines an optimal ISD for each deployment strategy as $D_{k, o p t}=\min \left(D_{k}^{*}, \hat{D_{k}}\right)$, where $\hat{D_{k}}$ represents the maximum ISD that can be selected to fulfill the certain area throughput target.

Considering this methodology, we illustrate the relationship between area power consumption and optimum ISD for varying area throughput targets in Fig. 3 where 70 percent of the users are assumed to be indoors, i.e., $I_{p}=0.7$. The results show that femtocell deployment significantly reduce the need 


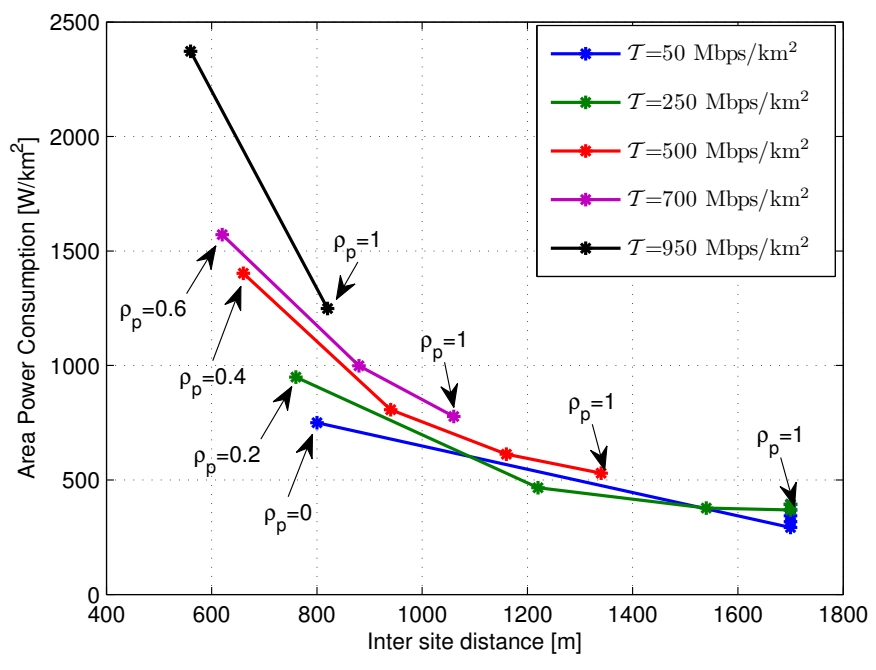

Fig. 3. Relationship between area power consumption, inter site distance and femto penetration rate for $I_{p}=0.7$.

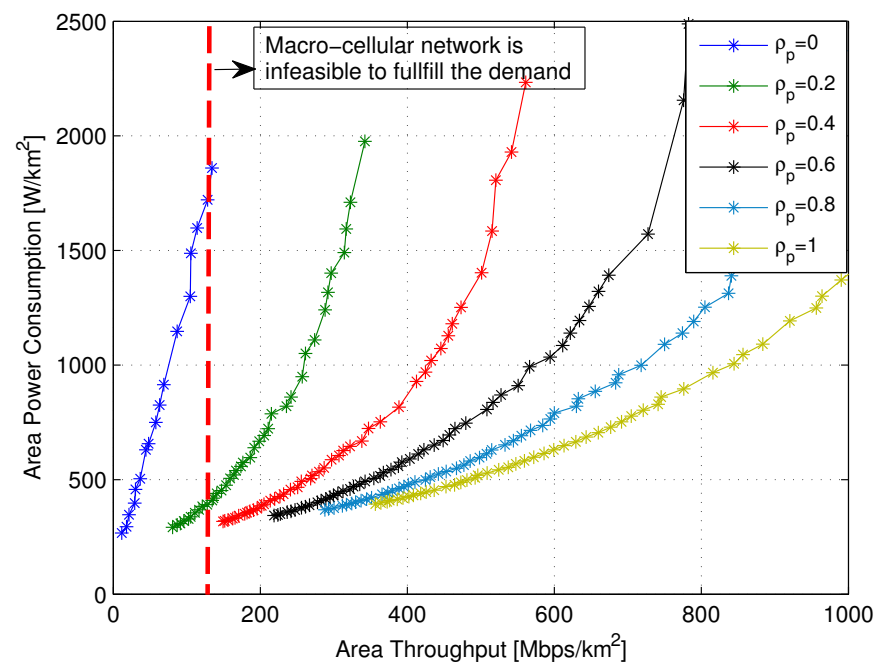

Fig. 4. Area power consumption vs. area throughput requirement for different femto penetration rate $\left(I_{p}=0.7\right)$.

for densification of the macro-cellular network for a given area throughput requirement which result in lower area power consumption. It is worthwhile noting that this optimum densification level decreases with femto penetration rate $\rho_{p}$ since more traffic is offloaded to femtocell network. It may come no surprise that higher capacity demands lower the optimum ISD and favors for smaller cells which is the main reason for the rapid increase in area power consumption with area throughput for each deployment strategy as depicted in Fig. 4. Note that besides its low energy efficiency figures, macro-only networks seem to become infeasible to satisfy the expected demand in dense urban areas for 2020 [20]. On the other hand, joint macro-femto deployment with varying $\rho_{p}$ extends the feasibility region with a relatively lower increase in area power consumption and thus appear to a promising solution to fulfill the medium and high area throughput requirements, i.e., $\mathcal{T} \geq 150 \mathrm{Mbps} / \mathrm{km}^{2}$ in an energy efficient manner.

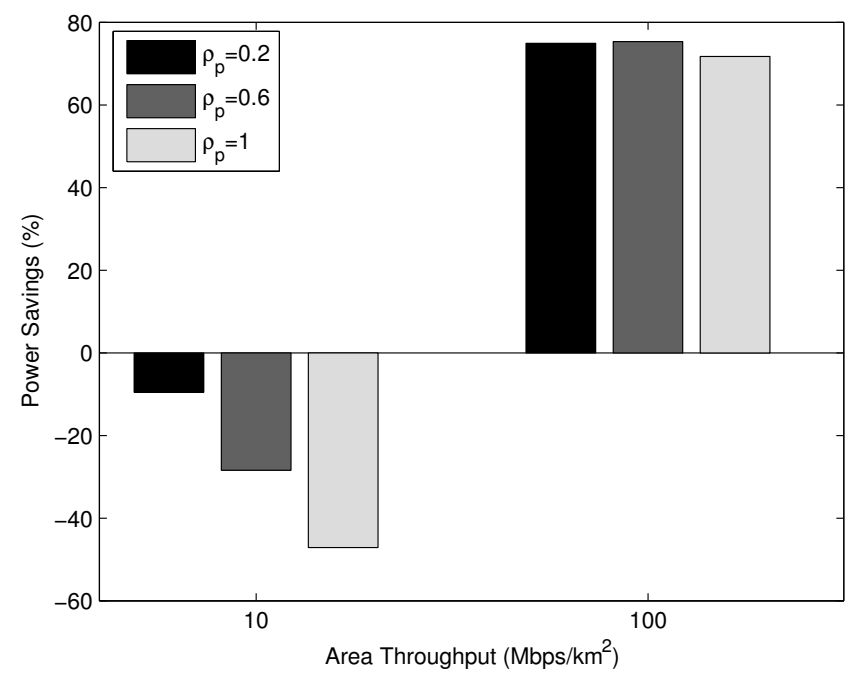

Fig. 5. Power savings compared to baseline scenario where all the users are served by the macro-cellular network $\left(I_{p}=0.5\right)$.

In order to quantify the impact of femtocell deployment on area power consumption in wireless access networks, we considered different area throughput targets from the feasibility region of the baseline system and calculated the power savings (expressed in percentage) compared to macro-only network for different femto penetration rates as shown in Fig. 5. It can be seen that for very low capacity demand, i.e., $\mathcal{T}=10 \mathrm{Mbps} / \mathrm{km}^{2}$, area power consumption increases linearly with the femto penetration rate. It is due to the fact that here the network is constrained by coverage, i.e., the required macro BS density for coverage purpose is enough to fulfill the area throughput requirement. Therefore, the deployment of additional femto BSs creates capacity over-provisioning with increase in area power consumption. However, rapidly growing demand for capacity reverses the situation and favors with the femtocell deployment which brings up to $75 \%$ savings compared to macro-only network for $\mathcal{T}=100 \mathrm{Mbps} / \mathrm{km}^{2}$ which is the expected traffic density in dense urban areas in 2015. Another notable result is that power savings via femtocell deployment with low femto penetration rate, e.g., $\rho_{p}=0.2$ is higher compared to dense femto deployment, i.e., $\rho_{p}=1$, when the target area throughput is $\mathcal{T}=100 \mathrm{Mbps} / \mathrm{km}^{2}$. This is because, for the latter case the reduced density of macrocellular network can not compensate the additional power consumption of femto BSs, leading to lower energy efficiency.

Fig. 6 illustrates the impact of the fraction of the indoor users $I_{p}$ on area power consumption for different deployment strategies, i.e, $\rho_{p} \in\{0,1\}$, considering low and high area throughput requirements. It has been observed that $I_{p}$ has a minor impact on the energy savings through femtocell deployment when the capacity demand is low. Another notable result is macro-only network $(\rho=0)$ is infeasible to fulfill $\mathcal{T}=500 \mathrm{Mbps} / \mathrm{km}^{2}$ regardless of user distribution. On the other hand, the fraction of the indoor users has a significant impact on the area power consumption of joint macro+femto deployment where around $40 \%$ savings is feasible as the more traffic generated in indoors for high capacity demand region. 


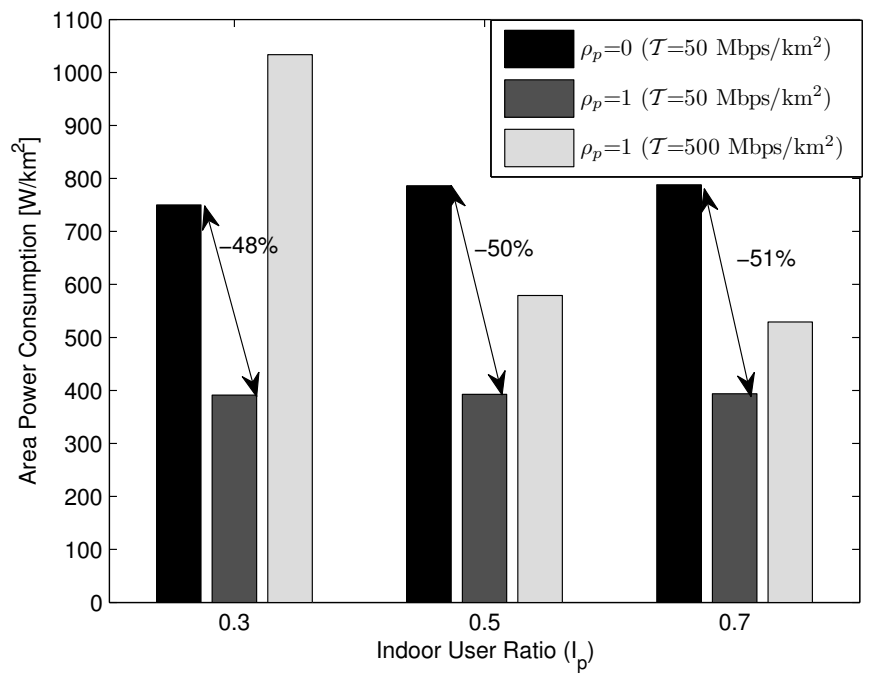

Fig. 6. Area power consumption as a function of indoor user ratio for low and high area throughput targets.

In summary, the observation in Fig. 5 and Fig. 6 indicate that femtocells have the potential to provide an energy efficient solution to cope with future traffic growth.

Note that, for simplicity of analysis, we consider random deployment which is not optimized according to spatial traffic demand. Therefore, a higher energy efficiency gain can be expected if more careful deployment has been achieved.

\section{CONCLUSION}

In this paper, we investigated the impact of femtocell deployment with varying density on the energy efficiency of wireless access networks. We demonstrated how different area throughput targets and percentage of indoor users affect the area power consumption taking into account the impact of backhaul. The results show that traditional macro-cellular networks achieves the minimum area power consumption only when the capacity requirement is very low. However, we observe that they quickly lose their efficiency with the increasing demand for high data rate and even becomes infeasible to fulfill the requirement. On the other hand, cochannel deployment of femto BSs under the macro-cellular coverage is shown to increase the area spectral efficiency due to high spectral frequency reuse and minor mutual interference between macro- and femtocells with a relatively lower increase in power consumption. This results in significant energy savings compared to macro-only network for medium and high capacity demand where the gain increases up to $75 \%$ as more traffic is offloaded to femtocells. Considering the fact that orders of magnitude of more mobile data traffic will be generated at indoors in the near future, blasting the signals over the walls is shown to be neither energy efficient nor feasible to satisfy the growing capacity demand.

\section{REFERENCES}

[1] S. Tombaz, A. Västberg, and J. Zander, "Energy and cost efficient ultra high capacity wireless access," IEEE Wireless Communications Magazine, vol. 18, no. 5, pp. 18-24, Oct. 2011.
[2] "Cisco visual networking index: Global mobile data traffic forecast update, 2010-2015,” Cisco Inc., Tech. Rep., February 2011.

[3] Z. Hasan, H. Boostanimehr, and V. Bhargava, "Green cellular networks: A survey, some research issues and challenges," IEEE Communications Surveys Tutorials, vol. 13, no. 4, pp. 524 -540, quarter 2011.

[4] L. Correia, D. Zeller, O. Blume, D. Ferling, Y. Jading, I. Gòdor, G. Auer, and L. Van Der Perre, "Challenges and enabling technologies for energy aware mobile radio networks," IEEE Communications Magazine, vol. 48, no. 11, pp. 66-72, Nov. 2010.

[5] K. Dufková, M. Bjelica, B. Moon, L. Kencl, and J. L. Boudec, "Energy savings for cellular network with evaluation of impact on data traffic performance," in Proc. of European Wireless Conference, Lucca, Italy, Apr. 2010.

[6] X. Wang, A. Vasilakos, M. Chen, Y. Liu, and T. Kwon, "A survey of green mobile metworks: Opportunities and challenges," ACM/Springer Mobile Networks and Applications (MONET), vol. 17, no. 1, pp. 4-20, Feb. 2012.

[7] J. Hoydis, M. Kobayashi, and M. Debbah, "Green small-cell networks," IEEE Vehicular Technology Magazine, vol. 6, no. 1, pp. 37 -43, March 2011.

[8] C. Han, T. Harrold, S. Armour, I. Krikidis, S. Videv, P. Grant, H. Haas, J. Thompson, I. Ku, C. Wang, T. Le, M. Nakhai, J. Zhang, and L. Hanzo, "Green radio: radio techniques to enable energy-efficient wireless networks," IEEE Communications Magazine, vol. 49, no. 6, pp. 46-54, June 2011.

[9] C. Khirallah, J. Thompson, and H. Rashvand, "Energy and cost impacts of relay and femtocell deployments in long-term-evolution advanced," IET Communications, vol. 5, no. 18, pp. 2617 -2628, Feb. 2011.

[10] J. Andrews, H. Claussen, M. Dohler, S. Rangan, and M. Reed, "Femtocells: Past, present, and future," IEEE J. Sel. Areas Commun., vol. 30, no. 3, pp. 497-508, Apr. 2012.

[11] H. Claussen, "Co-channel operation of macro- and femtocells in a hierarchical cell structure," International Journal of Wireless Information Networks, vol. 15, no. 3-4, pp. 137-147, Dec. 2008.

[12] K. Dufková, J. Le Boudec, M. Popović, M. Bjelica, R. Khalili, and L. Kencl, "Energy consumption comparison between macro-micro and public femto deployment in a plausible LTE network," in Proc. of Int. Conf. on Energy-Efficient Computing and Networking, Apr. 2011

[13] H. Claussen, L. Ho, and F. Pivit, "Effects of joint macrocell and residential picocell deployment on the network energy efficiency," in Proc. of IEEE Personal, Indoor and Mobile Radio Commun. (PIMRC), Cannes, France, Sept. 2008.

[14] M. Wang, X. Zhu, Z. Zeng, S. Wan, and W. Li, "System performance analysis of OFDMA-based femtocell networks," in Proc. of IET Int. Conf. on Communication Technology and Application(ICCTA), Oct. 2011.

[15] F. Cao and Z. Fan, "The tradeoff between energy efficiency and system performance of femtocell deployment," in Proc. of Int. Symp. on Wireless Communiaction Systems (ISWCS), York, UK, Sept. 2010.

[16] J. Gambini and U. Spagnolini, "Wireless over cable for energy-efficient femtocell systems," in Proc. of IEEE Global Comm. Conf. Workshops (GLOBECOM Wkshps), Miami, USA, Dec. 2010.

[17] A. D. Domenico, E. C. Strinati, and A. Duda, "Ghost femtocells: an energy-efficient radio resource management scheme for two-tier cellular networks," in Proc. of European Wireless Conference, Vienna, Austria, Apr. 2011.

[18] I. Ashraf, F. Boccardi, and L. Ho, "SLEEP mode techniques for small cell deployments," IEEE Communications Magazine, vol. 49, no. 8, pp. 72-79, Aug. 2011.

[19] T. S. G. R. A. Network, "TR 36.814-Further Advancements for EUTRA: Physical Layer Aspects (Release 9)," 3rd Generation Partnership Project Tech. Rep., Tech. Rep., 2009.

[20] G. Auer, V. Giannini, C. Desset, I. Godor, P. Skillermark, M. Olsson, M. Imran, D. Sabella, M. Gonzalez, O. Blume, and A. Fehske, "How much energy is needed to run a wireless network?" IEEE Wireless Communications Magazine, vol. 18, no. 5, pp. 40-49, Oct. 2011.

[21] S. Tombaz, P. Monti, K. Wang, A. Västberg, M. Forzati, and J. Zander, "Impact of backhauling bower consumption on the deployment of heterogeneous mobile networks," in Proc. of IEEE Global Comm. Conf. (GLOBECOM), Houston, USA, Dec. 2011.

[22] EARTH Deliverable D2.2, "Energy efficiency analysis of the reference systems, areas of improvements and target breakdown," Jan. 2012.

[23] F. Richter, A. J. Fehske, P. Marsch, and G. Fettweis, "Traffic demand and energy efficiency in heterogeneous cellular mobile radio networks," in Proc. of IEEE Vehic. Technol. Conf. (VTC Spring), Taipei, Taiwan, May 2010. 